\title{
PACAP signaling to DREAM: A cAMP-dependent pathway that regulates cortical astrogliogenesis
}

\author{
Mario Vallejo \\ Instituto de Investigaciones Biomédicas Alberto Sols, Consejo Superior de \\ Investigaciones Científicas /Universidad Autónoma de Madrid, Spain.
}

Address correspondence to:

Mario Vallejo, M.D., Ph.D.

Instituto de Investigaciones Biomedicas Alberto Sols

Calle Arturo Duperier 4

28029 Madrid, Spain

Tel.: 34-91-585 4480

Fax: 34-91-585 4401

Email: mvallejo@iib.uam.es 


\begin{abstract}
Astrocytes constitute a very abundant cell type in the mammalian central nervous system, and play critical roles in brain function. During development, astrocytes are generated from neural progenitor cells only after these cells have generated neurons. This so called gliogenic switch is tightly regulated by intrinsic factors that inhibit the generation of astrocytes during the neurogenic period. Once neural progenitors acquire gliogenic competence, they differentiate into astrocytes in response to specific extracellular signals. Some of these signals are delivered by neurotrophic cytokines via activation of the gp130-JAK-STAT system, whereas others depend on the activity of pituitary adenylate cyclase-activating polypeptide (PACAP) on specific PAC1 receptors that stimulate the production of cAMP. This results in the activation of the small GTPases Rap1 and Ras, and in the cAMP-dependent entry of extracellular calcium into the cell. Calcium, in turn, stimulates the transcription factor DREAM, which is bound to specific sites of the promoter of the glial fibrillary acidic protein gene, stimulating its expression during astrocyte differentiation. Lack of DREAM in vivo results in alterations in the number of neurons and astrocytes generated during development. Thus, the PACAP-cAMP-Ca ${ }^{2+}$-DREAM signaling cascade constitutes an important pathway to activate glial-specific gene expression during astrocyte differentiation.
\end{abstract}




\section{Introduction}

Among glial cells, astrocytes constitute the most abundant cell type in the central nervous system (Bass et al., 1971; Nedergaard et al., 2003). In recent years a large body of data has accumulated in favor of the notion that these glial cells participate actively in the physiological processes of the brain by establishing a reciprocal relationship with neurons (Barres, 2008; Theodosis et al., 2008). This relationship is not only important to ensure selectively the availability of nutrients through the blood brain barrier, but also for the performance of important tasks that are essential for the correct function and survival of neurons. Thus, astrocytes are active participants in synaptic transmission and regulate intercellular communication processes (Haydon and Carmignoto, 2006; Perea and Araque, 2007), they prevent neurotoxicity from excitatory neurotransmitters and other compounds (Ashner et al., 1999; Maragakis and Rothstein, 2004), and they produce trophic factors that are essential for neuronal differentiation and survival (Gash et al., 1998; Blondel et al., 2000). Interestingly, in some brain areas astrocytes themselves can give rise to neurons (Seri et al., 2001). In addition, astrocytes appear to be an important element in the development of certain diseases (Araque, 2006; Chen et al., 2007; Lepore et al., 2008), and mutations in the gene encoding glial fibrillary acidic protein (GFAP), an intermediate filament protein characteristic of these cells, are associated with Alexander's disease, a rare but devastating disorder of the central nervous system (Quinlan et al., 2007).

\section{Generation of astrocytes during brain development}

The mechanisms by which astrocytes originate from neural precursors during embryonic development have received much attention in recent years. It is now well established that during gestation the generation of astrocytes occurs at a relatively late 
stage, only after the generation of neurons has been practically completed, and proceeds postnatally. In rodents, neurogenesis spans approximately from embryonic day 12 (E12) to E18, whereas astrocytogenesis starts at around E17 and peaks a few days after birth. Importantly, both neurons and astrocytes arise in the developing neuroepithelium from the same progenitor cells, which appear to coincide with radial glial cells (Pinto and Gotz, 2007). Therefore, at a specific time during development progenitor cells are instructed to finish with the generation of neurons and to acquire a capacity to generate astrocytes.

This cellular switch from neurogenesis to gliogenesis is tightly regulated at different levels. One level of regulation involves the active inhibition of gliogenesis during the neurogenic period, so that precursors are unable to generate astrocytes even in the presence of astrogliogenic signals. One effective mechanism of inhibition consists on the methylation of astrocyte-specific genes leading to their inactivation in early progenitor cells (Takizawa et al., 2001; Namihira et al., 2004; Fan et al., 2005). In addition, inhibition of glia-specific genes in neuronal progenitors involves epigenetic silencing by chromatin remodeling (Song and Ghosh, 2004), as well as transcriptional repression (Hermanson et al., 2002; Angelastro et al., 2005). Interestingly, it has been found that transcription factors of the basic-helix-loop-helix family that promote the generation of neurons from progenitor cells, actively inhibit the expression of glialspecific genes, thus preventing gliogenesis (Cai et al., 2000; Nieto et al., 2001; Sun et al., 2001).

Neural progenitors gain competence to generate astrocytes due to the activity of growth factors such as basic fibroblast growth factor and epidermal growth factor (Qian et al., 1997; Viti et al., 2003; Song and Ghosh, 2004). This gain of competence allows them to respond to specific gliogenic signals acting at the extracellular level. 
Particularly important in this regard are signals provided by neurotrophic cytokines such as ciliary neurotrophic factor (CNTF), leukemia inhibitory factor (LIF) and cardiotrophin-1 (CT-1) (Johe et al., 1996; Bonni et al., 1997; Rajan and McKay, 1998; Barnabé-Heider et al., 2005). All of these cytokines activate heterodimeric cell surface receptors composed of two subunits named LIFR $\beta$ and gp130, which in turn activate members of the JAK family of tyrosine kinases, that result in the phosphorylation and nuclear translocation of signal transducer and activator of transcription (STAT) proteins (Schindler et al., 2007). In neural progenitors, two of these proteins, STAT1 and STAT3 act on specific sites in the promoters of the astroglial genes GFAP and $S 100 \beta$ to stimulate their transcription during the astrocyte differentiation process. In vivo studies carried out using genetically modified mice indicate that the gp130-JAK-STAT system is required for the generation of astrocytes, and that CT-1 provides a key regulatory signal for the activation of this system at the onset of the astrogliogenic period (Bugga et al., 1998; Koblar et al., 1998; Nakashima et al., 1999b; Barnabé-Heider et al., 2005).

Neural progenitors also respond to different neurotrophic factors from the bone morphogenetic proteins (BMP) family to generate astrocytes. In this case, BMP2 or BMP4 act on heterotrimeric receptors, which activate Smad transcription factors. These in turn, interact with activated STAT proteins to synergistically stimulate transcription of glial specific genes during astrocyte differentiation (Gross et al., 1996; Mehler et al., 1997; Mabie et al., 1999; Nakashima et al., 1999a).

\section{Identification of PACAP as a cAMP-dependent signal for the generation of astrocytes}

Apart from signals generated by diffusible factors acting on kinase-dependent receptors as discussed above, recent studies indicate that seven transmembrane domain 
G-protein-coupled receptors activated by pituitary adenylate cyclase-activating polypeptide (PACAP) can trigger the differentiation of astrocytes via a mechanism that requires the production of intracellular cAMP (McManus et al., 1999; Vallejo and Vallejo, 2002; Cebolla et al., 2008).

The studies that led to the identification of PACAP as a neurotrophic factor capable of triggering the differentiation of astrocytes from cortical precursors were initiated with the observation that stimulation of the cAMP-dependent signaling pathway in primary cultures of neuroepithelial progenitors obtained from rat fetal cortex results in the differentiation of astrocytes after exit from the cell cycle (McManus et al., 1999). This response observed after stimulation of the cAMP-dependent pathway in cortical progenitors is specifically instructive, because stimulation of this second messenger pathway does not result in the differentiation of neurons or oligodendrocytes (McManus et al., 1999), although these cells retain the capacity to differentiate into neurons in response to an appropriate stimulus such as BDNF (Lastres-Becker et al., 2008). After this initial observation, a search for an extracellular ligand that could mimic the effect of cAMP was initiated.

As a putative role for monoamine neurotransmitters had been suggested in the developing brain (Levitt et al., 1997; Cameron et al., 1998), and these neurotransmitters act by binding to G-protein-coupled receptors that can activate the synthesis of cAMP, they became obvious candidates for the observed cAMP-dependent astrocytogenic response. Indeed, exposure of primary fetal cortical progenitors to monoamines results in phosphorylation of CREB, indicating that these cells express appropriate functional receptors. However, these experiments showed that these cells do not differentiate into astrocytes when exposed to dopamine, noradrenaline or serotonine, as assessed by changes in morphology and expression of GFAP (Vallejo and Vallejo, 2002). 
Having ruled out monoamines, it was considered that certain neuropeptides acting on specific G-protein-coupled receptors could trigger the differentiation of astrocytes from cortical precursors. Because of the known activity of PACAP to stimulate adenylate cyclase in neural cells, this peptide and the closely related vasoactive intestinal polypeptide (VIP) were tested to investigate whether they are able to induce astrocyte differentiation.

Both PACAP and VIP can bind with similar affinities to two specific receptors known as VPAC1 and VPAC2. In addition, PACAP recognizes a third receptor known as PAC1, for which VIP exhibits a comparatively low affinity (Harmar et al., 1998). All of these receptors stimulate adenylate cyclase activity, although PAC1 receptors can also stimulate phospholipase $\mathrm{C}$, and both peptides have been reported to increase intracellular levels of cAMP in a variety of different cell types.

Experiments carried out with primary cultures of rat cortical progenitor cells demonstrated that PACAP, but not VIP, is able to promote their differentiation into astrocytes (Vallejo and Vallejo, 2002). The observed lack of effect of VIP and the specificity of PACAP is due to the circumstance that cortical neural progenitors express relatively high levels of the PAC1 receptor, but not VPAC1 or VPAC2 receptors. However, different isoforms of the PAC1 receptor generated by alternative splicing exist, and each one of these isoforms uses different signal transduction mechanisms. This is due to different configurations that may be adopted by the third intracellular loop of the receptor, which contains the domain that interacts directly with G-proteins to activate adenylate cyclase. Alternative splicing may result in the inclusion in this intracellular loop of one or two small cassettes called Hip and Hop, encoded by two different exons. This favors coupling of the receptor to the polyphosphoinositidespecific phospholipase C-dependent signaling pathway (Spengler et al., 1993; Nicot and 
DiCicco-Bloom, 2001). In addition, another PAC1 receptor isoform is able to stimulate calcium entry directly through the activation of an L-type calcium channel (Chatterjee et al., 1996). Therefore, the observation that PACAP is able to specifically mimic the astrocytogenic response of forskolin or $8 \mathrm{Br}-\mathrm{cAMP}$ in cortical progenitors does not necessarily mean that this neuropeptide is using a cAMP-dependent mechanism to produce this effect.

To clarify this issue, several lines of experimentation were undertaken. First, it was determined that the most abundant isoform of the PAC1 receptor expressed in rat cortical progenitor cells is the short isoform that couples to the G protein-adenylate cyclase system. Relatively small amounts of PAC1 receptor containing the Hop cassette, and even smaller amounts of the isoform containing the Hip cassette were also detected, but interestingly these disappeared upon receptor activation with PACAP or upon treatment of cells with the cAMP analog 8Br-cAMP at the expense of an increase in the expression of the short isoform of the receptor (Vallejo and Vallejo, 2002). Thus, the same stimulus that triggers the production of cAMP response also induces changes at the receptor level to maximize this response.

In a different set of experiments, it was determined that PACAP can stimulate the synthesis of cAMP in rat neural progenitor cells, whereas VIP is practically inactive. Importantly, astrocyte differentiation by PACAP can be inhibited by pretreatment with Rp-cAMPS, a specific antagonist of cAMP, indicating that the production of this second messenger is a critical step to initiate the differentiation response (Vallejo and Vallejo, 2002).

Thus, together these experiments demonstrated that PACAP can specifically induce astrocyte differentiation of primary cortical progenitor cells by acting on the short isoform of PAC1 receptors to stimulate intracellular cAMP production. 
Interestingly, only a relative short exposure of cells to PACAP (15-30 minutes) is required to trigger the astrocytogenic response, which can be evident even 5 days after peptide treatment by morphological changes and by the stimulation of GFAP expression. Thus, as it is the case with other neurotrophic factors inducing neuronal or glial differentiation (Gross et al., 1996; Williams et al., 1997; Park et al., 1999), PACAP appears to initiate the response by stimulating cAMP production, but then other intracellular mechanisms take over to promote differentiation.

Watanabe et al. (2006), following studies carried out by the same group (Ohno et al., 2005), confirmed that PACAP is indeed capable to induce astrocyte differentiation by acting on PAC1 receptors. However, they did not find evidence in favor of a role for cAMP in this response, but instead proposed an involvement of phospholipase $\mathrm{C}$ and protein kinase $\mathrm{C}$. These authors used relatively early (E14.5) mouse neural progenitor cells, as opposed to the E17 neural progenitors prepared from rat fetuses used in the experiments described above. In addition, relatively low concentrations of PACAP were used, and the differentiation of astrocytes took up to 5 days to be observed (Ohno et al., 2005; Watanabe et al., 2006). Interestingly, it has been found that activation of $\mathrm{PAC} 1$ receptors in mouse neural precursors can generate both intracellular cAMP accumulation and activation of phospholipase $\mathrm{C}$-mediated signaling leading to an increase in intracellular calcium, although this latter response appears to be related to proliferation of glial precursors rather than to differentiation (Nishimoto et al., 2007). Thus, differences in the species and experimental conditions may reflect the complex mechanisms activated by PACAP in the developing brain.

Both PACAP and the PAC1 receptor have been described to be present in the brain throughout development and postnatally (Suh et al., 2001; Nishimoto et al., 2007; Watanabe et al., 2007). A role for PACAP in the regulation of astrogliogenesis has been 
proposed based on studies carried out by different investigators (Zupan et al., 1998; Vallejo and Vallejo, 2002; Ohno et al., 2005; Cebolla and Vallejo, 2006; Watanabe et al., 2006; Nishimoto et al., 2007; Cebolla et al., 2008; Lastres-Becker et al., 2008). However, it is clear that the role of PACAP during development is complex, because at different times during development it exerts different actions. Thus, independently of its activity as an astrogliogenic signal, PACAP acts as a potent antimitotic factor on relatively early neural progenitors and favors neuronal differentiation, whereas on late progenitors it regulates the generation of oligodendrocytes (Lee et al., 2001; Nicot and

DiCicco-Bloom, 2001; Suh et al., 2001; Carey et al., 2002; Cazillis et al., 2004). This variety of responses at different times during development indicates that PACAP is an important neurotrophic factor whose activity is tightly regulated at different levels, including the specific type of receptor isoform expressed by neural progenitors, the different signal transduction pathways used by these receptors, the possible interaction of these pathways with molecules of signaling pathways activated by other neurotrophic factors, the type of transcription factors activated that act on specific target genes, and possibly the degree of responsiveness of these genes which may depend on epigenetic modifications. In turn, each one of these aspects may vary in different cells throughout development, thus providing an explanation for the high degree of versatility with which PACAP exerts different functions depending on the developmental stage and the type of the target cells on which it acts.

\section{Intracellular cAMP-dependent signaling triggered by PACAP in cortical precursors}

As the differentiation of astrocytes from progenitor cells is accompanied by stimulation of the expression of the gene encoding GFAP, monitoring the 
transcriptional activity of the GFAP promoter driving a reporter gene such as luciferase provided a useful approach to investigate the intracellular signaling pathways activated by PACAP during astrocyte differentiation from neural progenitors.

Activation of protein kinase A (PKA) was initially considered to be an obvious mechanism by which PACAP could stimulate GFAP gene expression and trigger astrocyte differentiation. This notion was supported by three lines of evidence: First, the astrogliogenic effect of PACAP on cortical progenitors can be inhibited by the cAMP antagonist Rp-cAMPS; second, treatment of cortical progenitors with PACAP results in phosphorylation of CREB, a well known substrate for PKA; and third, PACAP-induced CREB phosphorylation can be inhibited by pretreatment with the PKA inhibitor H89 (Lastres-Becker et al., 2008). However, it soon became clear that PKA is not involved, because $\mathrm{H} 89$ was not able to inhibit the stimulation of GFAP gene expression nor the differentiation of astrocytes induced by PACAP. Thus, although PACAP can activate PKA in cortical progenitors, this activation may be responsible for other effects such as inhibition of proliferation (Lelievre et al., 2002), but not for inducing the differentiation of these progenitors into astrocytes.

Besides PKA, cAMP can stimulate at least two additional intracellular signaling cascades by binding to guanine nucleotide exchange proteins such as Epac and CNrasGEF (Pham et al., 2000; Bos, 2003). In cortical progenitors, PACAP is able to stimulate both pathways (Lastres-Becker et al., 2008). In the first case, exposure of cells to PACAP results in the activation of Rap1, which acts as an effector for cAMPactivated Epac. In the second case, PACAP treatment leads to stimulation of Ras, an effect that can be inhibited by the cAMP-specific antagonist Rp-cAMPS. The activation of Rap1 and Ras by PACAP is not restricted to neural progenitors, since it has been 
observed in different types of neurons and astrocytes (Bouschet et al., 2003; Dasgupta et al., 2003; Romano et al., 2003; Obara et al., 2007).

The notion that stimulations of these pathways are involved in triggering astrocyte differentiation by PACAP is supported by the observations that Rap1N17 or RasN17, which act as dominant negative inhibitors of Rap1 and Ras, respectively (Vossler et al., 1997; Matallanas et al., 2003), are able to decrease the stimulation of the GFAP gene promoter elicited by PACAP in cortical precursor cells. That Ras is stimulated in a cAMP-dependent manner was demonstrated by the observation that PACAP is unable to activate Ras in the presence of Rp-cAMPS, and that GFAP promoter stimulation by the stable cAMP analog $8 \mathrm{Br}-\mathrm{cAMP}$ is significantly reduced in the presence of the dominant negative inhibitor RasN17 (Lastres-Becker et al., 2008).

Interestingly, stimulation of either the Epac-Rap1 or the cAMP-Ras pathway in isolation by using the selective activator of Epac 8CPT-2-O-Me-cAMP (Enserink et al., 2002), or by expressing RasV12, a constitutively active form of Ras, is not sufficient to stimulate GFAP gene expression. For this to occur, both signaling cascades need to be concomitantly stimulated, consistent with the observations that coexpression of both RapN17 and RasN17 completely inhibits GFAP promoter stimulation by PACAP, and that combined knock down of Ras and Rap1 isoforms by siRNA prevents expression of GFAP after stimulation of cortical progenitors with PACAP (Lastres-Becker et al., 2008).

The data described above, obtained using primary cultures of cortical precursors in vitro are in agreement with those observed in vivo (Apicelli et al., 2003). These authors generated transgenic mice expressing a constitutively active form of Rap1 under the control of the GFAP promoter, so that its expression is restricted to astrocytes from a time coinciding with astrogliogenesis at around E16. In these mice, astrocyte 
differentiation was not significantly altered, as the number of astrocytes generated was similar to that of control mice.

\section{Transcriptional stimulation of the GFAP promoter by PACAP-cAMP}

Studies to identify transcriptional mechanisms that activate and restrict the expression of the GFAP gene to astrocytes have been carried out both in cultured cells and in transgenic mice (Barnett et al., 1995; Bonni et al., 1997; Kahn et al., 1997; Rajan and McKay, 1998; Stone et al., 1998; Krohn et al., 1999; Nakashima et al., 1999a; Sun et al., 2001; Rajan et al., 2003; Lee et al., 2006; Lee et al., 2008).

Among the different transcription factors identified to date that regulate GFAP gene expression, some members of the NFI family appear to play a prominent role (Cebolla and Vallejo, 2006; Deneen et al., 2006; Gopalan et al., 2006). The NFI transcription factors comprise a family composed of at least four members with important functions during development (Gronostajski, 2000). Studies carried out in the developing spinal cord indicate that one member of this family, NFIA, is necessary for the gliogenic switch by which neural precursors inhibit their capacity to differentiate into neurons and acquire an astroglial phenotype (Deneen et al., 2006). In addition, NFI genes are important for the promotion of astrocyte differentiation, and their deletion results in decreased levels of GFAP expression (Das Neves et al., 1999; Shu et al., 2003; Steele-Perkins et al., 2005; Deneen et al., 2006).

In the brain of rat fetuses NFI occupies specific sites of the GFAP promoter in vivo, and therefore it is not surprising that the onset of expression of NFI in the developing cortex coincides with the onset of GFAP expression during astrogliogenesis at around E17 (Cebolla and Vallejo, 2006). In the absence of NFI binding to the GFAP gene, activation of the promoter by astrogliogenic factors such as PACAP or CNTF 
remains functional but is significantly impaired. NFI has been found to regulate transcription by presetting the chromatin for accessibility to other transcription factors (Belikov et al., 2004; Hebbar and Archer, 2007). Therefore, it is possible that NFI could be important in neural cells for maintaining the GFAP promoter in a favorable condition for its activation during astrocyte differentiation via a similar mechanism. This remains an important question to be addressed in future studies.

The stimulation of GFAP gene transcription by PACAP or by cAMP agonists even in the absence of NFI binding, although reduced, clearly indicates the existence of promoter elements that bind specific transcription factors different from NFI, which are activated by cAMP at the beginning of the astrocyte differentiation process. Analyses of the rat GFAP promoter in primary cultures of cortical progenitors led to the characterization of two regulatory elements bound by transcription factor DREAM, and to the demonstration that DREAM is the effector protein that mediates the transcriptional stimulation triggered by PACAP and cAMP at the onset of astrogliogenesis (Cebolla et al., 2008).

In addition, both PACAP and CNTF facilitate astrocytogenesis cooperatively, so that when both factors are present together, cells are generated with longer and more elaborate processes than those exposed to only one of these factors. This synergism does not occur in the absence of NFI binding to the GFAP promoter, suggesting that NFI is also important for the integration of different signaling pathways at the transcriptional level (Cebolla and Vallejo, 2006). The mechanism by which this integration resulting in synergism occurs is not known, but it is possible that it involves interactions of NFI with both STAT proteins and DREAM. In the first case, such type of interactions have been described in other cell types (Mukhopadhyay et al., 2001), whereas in the second, the NFI and DREAM binding sites in the rat and mouse GFAP 
promoter are located in such close proximity (nucleotides -79 to -67 and -62 to -59 , respectively, relative to the transcription initiation site) that direct interactions are indeed likely (Fig. 1). An analysis of the promoter sequences of the GFAP gene from different species using the UCSC Genome Bioinformatics database (http://genome.ucsc.edu) shows that the NFI binding site is well conserved among different species including rat, mouse, dog, cow and human. The adjacent DREAM binding site is conserved in rat and mouse, but is divergent in other species. However, a conserved DREAM binding site is present in the human GFAP promoter approximately 160 base pairs upstream from the NFI site.

\section{DREAM transactivates the GFAP promoter in response to a cAMP-dependent increase in intracellular calcium}

DREAM (Downstream Regulatory Element Antagonist Modulator) was initially identified as a repressor protein bound to regulatory elements located downstream from the transcription initiation site of the prodynorphin and c-fos genes (Carrion et al., 1999; Ledo et al., 2000). In cortical progenitor cells, DREAM is bound to at least two sites in the GFAP promoter even before they are induced to differentiate by PACAP, and once GFAP expression is initiated and astrocytes are generated DREAM remains bound. Indeed, the integrity of these two sites, and therefore binding of DREAM to the GFAP gene appears to be essential for promoter activity (Cebolla et al., 2008).

That DREAM is essential for mediating PACAP-induced stimulation of GFAP expression and astrocyte differentiation was initially indicated by the observation that cortical progenitor cells derived from DREAM-deficient mice fail to differentiate into astrocytes in response to PACAP, although they can still generate astrocytes in response to $\mathrm{CNTF}$, indicating that the PACAP-DREAM signaling pathway works independently 
of the gp130-JAK-STAT signaling pathway used by neurotrophic cytokines (Cebolla et al., 2008).

DREAM can be stimulated by interacting with the transcription factor $\alpha C R E M$ after phosphorylation of this factor by PKA following activation by cAMP, or by direct binding of calcium ions to specific domains (Carrion et al., 1999; Ledo et al., 2000). In concert with the previous observation that PKA activation is not involved in the generation of astrocytes induced by PACAP, cAMP-dependent interactions of $\alpha$ CREM and DREAM are not involved in stimulation of GFAP expression by PACAP. On the contrary, PACAP/cAMP-induced GFAP expression in cortical progenitors requires the integrity of calcium-binding domains in DREAM (Cebolla et al., 2008), adding calcium as an additional component of the signaling cascade that links PACAP activation of PAC1 receptors with GFAP promoter stimulation.

PACAP can increase intracellular concentrations of calcium by a variety of mechanisms (Przywara et al., 1996; Osipenko et al., 2000; Morita et al., 2002; Liu et al., 2003; Payet et al., 2003; Nishimoto et al., 2007). In rat cortical progenitors, this rise in calcium is due to entry from the extracellular milieu. As observed in other cell types (Przywara et al., 1996; Osipenko et al., 2000), PACAP-induced entry of calcium into the cells is mediated by cAMP, because pretreatment with the cAMP antagonist RpcAMPS completely blocks this response (Cebolla et al., 2008).

The mechanism by which DREAM transactivates the GFAP promoter in response to calcium is unknown, but it is likely that it involves changes in protein conformation (Carrion et al., 1999; Osawa et al., 2001; Osawa et al., 2005). DREAM binds to DNA as a tetramer and ion-induced conformational changes result in the generation of calcium-bound DREAM dimers that normally do not bind DNA (Osawa et al., 2001; Osawa et al., 2005; Lusin et al., 2008). However, southwestern analysis has 
shown that DREAM dimers can bind DNA (Carrion et al., 1999), and in the case of the GFAP promoter, this binding could be stabilized by interactions with other proteins bound in close proximity. In this regard, it is important to note that NFI occupies a site in the GFAP promoter located adjacent to one of the DREAM binding sites, and that binding of NFI to this site is important for GFAP expression during astrocyte differentiation (Cebolla and Vallejo, 2006). Thus, it is possible that DREAM is bound to the GFAP promoter as part of a multiprotein complex including NFI, and that calcium binding to DREAM induces conformational changes that favor positive interactions with coactivators or with other transcriptionally active proteins. Indeed, it has been indicated that the mechanism by which DREAM acts on target genes bearing DREAM binding sites upstream, rather than downstream of the TATA box, may be different and may involve interactions with other transcription factors (Rivas et al., 2004; Gomez-Villafuertes et al., 2005; Scsucova et al., 2005).

In this regard, and in view of the observed capacity of DREAM to interact with other transcription factors (Ledo et al., 2000; Rivas et al., 2004; Scsucova et al., 2005), it is important to distinguish between the effects on GFAP promoter activation obtained experimentally after mutating either of the two DREAM binding sites in vitro, and the effects obtained on endogenous GFAP expression after inactivating the expression of DREAM in vivo. Thus, whereas the integrity of DREAM binding sites is essential for GFAP promoter activity in vitro, in cells lacking DREAM the GFAP promoter can still be functional (Cebolla et al., 2008). One possible interpretation of these apparently conflicting observations is that DREAM may be engaged in interactions with NFI and/or with other unidentified transcription factors assembled in such a way that promoter activity requires the coordinated binding of the complex, as depicted schematically in figure 1. Mutation of the DREAM binding sites could result in the 
sequestration of the entire complex if DREAM is not given access to DNA (Fig. 1B). In contrast, in the absence of DREAM the remaining proteins could still be able to bind to their cognate sites on DNA and activate transcription (Fig. 1C). To validate or reject this model, studies to investigate whether DREAM interacts with NFI, or with STAT proteins or other transcription factors on the GFAP promoter in differentiating astrocytes will be required.

\section{DREAM and the timing of the transition from neurogenesis to gliogenesis}

The physiological importance of DREAM in the mechanisms that regulate the generation of astrocytes during development was indicated by the observation that the number of astrocytes present during the early postnatal gliogenic period in mice lacking DREAM is reduced (Cebolla et al., 2008). This reduction is probably a reflection of a defect in the relay of PACAP/cAMP/calcium-dependent signals to the transcription machinery assembled on the GFAP gene, and is consistent with previous studies showing reduced number of astrocytes in the offspring of rats treated with a PACAP receptor antagonist during late pregnancy (Zupan et al., 1998). However, this reduction is not permanent. From the seventh day of postnatal life, the number of astrocytes in DREAM-mutant mice rises to even surpass that of control mice (Cebolla et al., 2008). Therefore, lack of DREAM does not affect other astrocyte-generating signals that may act coordinately during development.

The mechanisms that regulate the cellular switch by virtue of which cortical progenitors inhibit their capacity to generate neurons and start generating astrocytes are tightly linked. Therefore, when gliogenesis is inhibited a concomitant increase in neurogenesis may be observed. The reason for this is that signals that activate gliogenesis are also actively involved in the inhibition of neurogenesis (Miller and 
Gauthier, 2007). It follows that if DREAM is also involved in repressing neurogenesis when astrogliogenesis is about to start, the initial decrease in the number of astrocytes in DREAM-deficient mice followed by the subsequent rise at a later stage could be interpreted as a delay in the onset of astrogliogenesis at the expense of maintaining neurogenesis for a longer period. Support for this interpretation is given by the observation that cortical progenitors of DREAM-mutant mice generate more neurons than those of control mice both in vitro and in vivo (Cebolla et al., 2008). But then, why are more astrocytes generated in the end?

The answer to this question may be related to the discovery that neurotrophic cytokines such as cardiotrophin-1 generated from neurons provide a powerful astrogliogenic signal (Barnabé-Heider et al., 2005). Thus, the proposed scenario would be that DREAM may have two different functions: on the one hand, it would inhibit neurogenesis at the end of the neurogenic period, and on the other hand, it would coordinately stimulate the generation of astrocytes from the same progenitor cells. This would explain that when DREAM is not present, neurogenesis would go on for a longer period of time generating an increased number of neurons at the expense of astrocytes. Later, neurotrophic cytokines secreted by these neurons would in turn act on the progenitors to inhibit neurogenesis and stimulate astrogliogenesis, and because of the initial overproduction of neurons these cytokines would be present in higher concentrations, thus resulting in a relative overproduction of astrocytes.

This model has two important implications. One is that lack of DREAM does not alter the gp130-JAK-STAT signaling pathway used by neurotrophic cytokines (Barnabé-Heider et al., 2005; Cebolla et al., 2008). The other is that during normal development, the astrogliogenic instructions delivered by the PACAP-cAMP-calciumDREAM system precede those delivered by neuron-generated neurotrophic cytokines. 
Of course, future studies are needed to confirm or reject these notions. In addition, although lack of DREAM appears to affect astrocyte development throughout the brain (Cebolla et al., 2008), it remains to be determined whether in different regions of the developing central nervous system the stimulation of GFAP promoter by DREAM is activated by PACAP, as it appears to be the case with cortical astrocytes, or by different cAMP-generating factors acting on neural progenitor cells.

\section{Conclusions}

Our understanding of the molecular mechanisms by which proliferating neuroepithelial progenitors differentiate into neurons and glial cells during brain development is increasing rapidly. The emerging scenario is one in which different types of processes acting at different levels, including epigenetic changes in chromatin, differential expression of transcription factors and specific interactions among them, as well as coordinated activation of intracellular signaling cascades triggered by factors acting on the cell membrane, are tightly regulated. The studies described in this review support the notion that the PACAP-cAMP-calcium-DREAM cascade constitutes an important signaling pathway that participates in the regulation of the gliogenic switch and the generation of astrocytes in the developing brain (Fig. 2).

Based on these studies emerging questions arise. For example, what is the mechanism by which NFI facilitates CNTF- and PACAP-induced GFAP expression? Does DREAM directly interact with NFI or STAT proteins? How does DREAM participate in the regulation of the number of neurons generated? Is DREAM involved in the stimulation of GFAP expression observed in reactive astrocytes? These questions open new avenues of investigations that will contribute to elucidate the complex mechanisms by which cellular diversity is generated in the mammalian brain. 


\section{Acknowledgements}

I would like to thank past and present members of my laboratory who generated published data discussed in this review, and Javier Perez for electronically drawing the figures. Research funding was provided by grants form the Spanish Ministry of Science and Innovation and from the Comunidad de Madrid. 


\section{REFERENCES}

Angelastro JM, Mason J, Ignatova TN, Kukekov VG, Stengren GB, Goldman JE, Greene LA (2005) Downregulation of activating transcription factor 5 is required for differentiation of neural progenitor cells into astrocytes. J Neurosci 25:3889-3899.

Apicelli AJ, Uhlmann EJ, Baldwin RL, Dong D, Nagy A, Guha A, Gutman DH (2003) Role of the Rap1 GTPase in astrocyte growth regulation. Glia 42:225-234.

Araque A (2006) Astrocyte-neuron signaling in the brain. Implications for disease. Curr Opin Investig Drugs 7:619-624.

Ashner M, Allen JW, Kimelberg HK, LoPachin RM, Streit WJ (1999) Glial cells in neurotoxicity development. Annu Rev Pharmacol Toxicol 39:151-173.

Barnabé-Heider F, Wasylnka JA, Fernandes KJL, Porsche C, Sendtner M, Kaplan DR, Miller FD (2005) Evidence that embryonic neurons regulate the onset of cortical gliogenesis via cardiotrophin-1. Neuron 48:253-265.

Barnett SC, Rosario M, Doyle A, Kilbey A, Lovatt A, Gillespie DAF (1995) Differential regulation of AP-1 and novel TRE-specific DNA-binding complexes during differentiation of oligodendrocyte-type-2-astrocyte (O-2A) progenitor cells. Development 121:3969-3977.

Barres BA (2008) The mistery and magic of glia: A perspective on their roles in health and disease. Neuron 60:430-440.

Bass NH, Hess HH, Pope A, Thalheimer C (1971) Quantitative cytoarchitectonic distribution of neurons, glia, and DNA in rat cerebral cortex. J. Comp. Neurol. 143:481-490.

Belikov S, Holmqvist PH, Astrand C, Wrange O (2004) Nuclear factor 1 and octamer transcription factor 1 binding preset the chromatin structure of the mouse 
mammary tumor virus promoter for hormone induction. J Biol Chem 279:49857-49867.

Blondel O, Collin C, McCarran WJ, Zhu S, Zamostiano R, Gozes I, Brenneman DE, McKay RDG (2000) A glia-derived signal regulating neuronal differentiation. J Neurosci 20:8012-8020.

Bonni A, Sun Y, Nadal-Vicens M, Bhatt A, Frank DA, Rozovsky I, Stahl N, Yancopoulos GD, Greenberg ME (1997) Regulation of gliogenesis in the central nervous system by the JAK-STAT signaling pathway. Science 278:477-483.

Bos JL (2003) Epac: a new cAMP target and new avenues in cAMP research. Nature Rev Mol Cell Biol 4:733-738.

Bouschet T, Perez V, Fernandez C, Bockaert J, Eychene A, Journot L (2003) Stimulation of the ERK pathway by GTP-loaded Rap1 requires the concomitant activation of Ras, protein kinase $\mathrm{C}$, and protein kinase $\mathrm{A}$ in neuronal cells. J Biol Chem 278:4778-4785.

Bugga L, Gadient RA, Kwan K, Stewart CL, Patterson PH (1998) Analysis of neuronal and glial phenotypes in brains of mice deficient in leukemia inhibitory factor. $\mathrm{J}$ Neurobiol 36:509-524.

Cai L, Morrow EM, Cepko CL (2000) Misexpression of basic helix-loop-helix genes in the murine cerebral cortex affects cell fate choices and neuronal survival. Development 127:3021-3030.

Cameron HA, Hazel TG, McKay RD (1998) Regulation of neurogenesis by growth factors and neurotransmitters. J Neurobiol 36:287-306.

Carey RG, Li B, DiCicco-Bloom E (2002) Pituitary adenylate cyclase activating polypeptide anti-mitogenic signaling in cerebral cortical progenitors is regulated by p57 ${ }^{\text {Kip2}}$-dependent CDK2 activity. J Neurosci 22:1583-1591. 
Carrion AM, Link WA, Ledo F, Mellstrom B, Naranjo JR (1999) DREAM is a $\mathrm{Ca}^{2+}$ regulated transcriptional repressor. Nature 398:80-84.

Cazillis M, Gonzalez BJ, Billardon C, Lombet A, Fraichard A, Samarut J, Gressens P, Vaudry H, Rostene W (2004) VIP and PACAP induce selective neuronal differentiation of mouse embryonic stem cells. Eur J Neurosci 19:798-808.

Cebolla B, Vallejo M (2006) Nuclear factor-I regulates glial fibrillary acidic protein gene expression in astrocytes differentiated from cortical precursor cells. J Neurochem 97:1057-1070.

Cebolla B, Fernández-Pérez A, Perea G, Araque A, Vallejo M (2008) DREAM mediates cAMP-dependent, $\mathrm{Ca}^{2+}$-induced stimulation of GFAP gene expression and regulates cortical astrogliogenesis. J Neurosci 28:6703-6713.

Chatterjee TK, Sharma RV, Fisher RA (1996) Molecular cloning of a novel variant of the pituitary adenylate cyclase-activating polypeptide (PACAP) receptor that stimulates calcium influx by activation of L-type calcium channels. J Biol Chem 217:32226-32232.

Chen G, Li HM, Chen YR, Gu XS, Duan S (2007) Decreased estradiol release from astrocytes contributes to the neurodegeneration in a mouse model of NiemannPick type C. Glia 15:1509-1518.

Das Neves G, Duchala CS, Tolentino-Silva F, Haxhiu MA, Colmenares C, Macklin WB, Campbell CE, Butz KG, Gronostajski RM (1999) Disruption of the murine nuclear factor I-A gene (Nfia) results in perinatal lethality, hydrocephalus, and agenesis of the corpus callosum. Proc Natl Acad Sci USA 96:11946-11951.

Dasgupta B, Dugan LL, Gutmann DH (2003) The neurofibromatosis 1 gene product neurofibromin regulates pituitary adenylate cyclase-activating polypeptidemediated signaling in astrocytes. J Neurosci 23:8948-8954. 
Deneen B, Ho R, Lucaszewicz A, Hochstim CJ, Gronostajski RM, Anderson DJ (2006) The transcription factor NFIA controls the onset of gliogenesis in the developing spinal cord. Neuron 52:953-968.

Enserink JM, Christensen AE, de Rooij J, van Triest M, Schwede F, Genieser HG, Doskeland SO, Blank JL, Bos JL (2002) A novel Epac-specific cAMP analogue demonstrates independent regulation of Rap1 and ERK. Nat Cell Biol 4:901906.

Fan G, Martinowich K, Chin MH, He F, Fouse SD, Hutnik L, Hattori D, Ge W, Shen Y, Wu H, Ten Hoeve J, Shuai K, Sun YE (2005) DNA methylation controls the timing of astrogliogenesis through regulation of JAK-STAT signaling. Development 132:3345-3356.

Gash DM, Zhang Z, Gerhardt G (1998) Neuroprotective and neurorestorative properties of GDNF. Ann Neurol 44:121-125.

Gomez-Villafuertes R, Torres B, Barrio J, Savignac M, Gabellimi N, Rizzato F, Pintado B, Gurtierrez-Adan A, Mellstrom B, Carafoli E, Naranjo JR (2005) Downstream regulatory element antagonist modulator regulates $\mathrm{Ca}^{2+}$ homeostasis and viability in cerebellar neurons. J Neurosci 25:10822-10830.

Gopalan SM, Wilczynska KM, Konik BS, Bryan L, Kordula T (2006) Nuclear factor-I$\mathrm{X}$ regulates astrocyte-specific expression of the alpha1-antichymotrypsin and glial fibrillary acidic protein genes. J Biol Chem 281:13126-13133.

Gronostajski RM (2000) Roles of the NF1/CTF gene family in transcription and development. Gene 249:31-45.

Gross RE, Mehler MF, Mabie PC, Zang Z, Santschi L, Kessler JA (1996) Bone morphogenetic proteins promote astroglial lineage commitment by mammalian subventricular zone progenitor cells. Neuron 17:595-606. 
Harmar AJ, Arimura A, Gozes I, Journot L, Laburthe M, Pisegna JR, Rawlings SR, Robberecht P, Said SI, Sreedharan SP, Wank SA, Waschek JA (1998) International Union of Pharmacology. XVIII. Nomenclature of receptors for vasoactive intestinal peptide and pituitary adenylate cyclase-activating polypeptide. Pharmacol Rev 50:265-270.

Haydon PG, Carmignoto G (2006) Astrocyte control of synaptic transmission and neurovascular coupling. Physiol Rev 86:1009-1031.

Hebbar PB, Archer TK (2007) Chromatin-dependent cooperativity between site-specific transcription factors in vivo. J Biol Chem 282:8284-8291.

Hermanson O, Jepsen K, Rosenfeld MG (2002) N-CoR controls differentiation of neural stem cells into astrocytes. Nature 419:934-939.

Johe KK, Hazel TG, Muller T, Dugich-Djordjevic MM, McKay RDG (1996) Single factors direct the differentiation of stem cells from the fetal and adult central nervous system. Genes Dev 10:3129-3140.

Kahn MA, Huang CJ, Caruso A, Barresi V, Nazarian R, Condorelli DF, De Vellis J (1997) Ciliary neurotrophic factor activates JAK/Stat signal transduction cascade and induces transcriptional expression of glial fibrillary acidic protein in glial cells. J Neurochem 68:1413-1423.

Koblar SA, Turnley AM, Classon BJ, Reid KL, Ware CB, Cheema SS, Murphy M, Bartlett PF (1998) Neural precursor differentiation into astrocytes requires signaling through the leukemia inhibitory factor receptor. Proc Natl Acad Sci USA 95:3178-3181.

Krohn K, Rozovsky I, Wals P, Teter B, Anderson CP, Finch CE (1999) Glial fibrillary acidic protein transcription responses to transforming growth factor- $\beta 1$ and 
interleukin-1 $\beta$ are mediated by a nuclear factor-1-like site in the near-upstream promoter. J Neurochem 72:1353-1361.

Lastres-Becker I, Fernández-Pérez A, Cebolla B, Vallejo M (2008) Pituitary adenylate cyclase-activating polypeptide stimulates glial fibrillary acidic protein gene expression in cortical precursor cells by activating Ras and Rap1. Mol Cell Neurosci 39:291-301.

Ledo F, Carrion AM, Link WA, Mellstrom B, Naranjo JR (2000) DREAM-alphaCREM interaction via leucine-charged domains derepresses downstream regulatory element-dependent transcription. Mol Cell Biol 20:9120-9126.

Lee M, Lelievre V, Zhao P, Torres M, Rodriguez W, Byun JY, Doshi S, Ioffe Y, Gupta G, de los Monteros AE, de Vellis J, Waschek J (2001) Pituitary adenylyl cyclase-activating polypeptide stimulates DNA synthesis but delays maturation of oligodendrocyte progenitors. J Neurosci 21:3849-3859.

Lee Y, Su M, Messing A, Brenner M (2006) Astrocyte heterogeneity revealed by expression of a GFAP-LacZ transgene. Glia 53:677-687.

Lee Y, Messing A, Su M, Brenner M (2008) GFAP promoter elements required for region-specific and astrocyte-specific expression. Glia 56:481-493.

Lelievre V, Hu Z, Byun JY, Ioffe Y, Waschek JA (2002) Fibroblast growth factor-2 converts PACAP growth action on embryonic hindbrain precursors from stimulation to inhibition. J Neurosci Res 67:566-573.

Lepore AL, Rauk B, Dejea C, Pardo AC, Rao MS, Rothstein JD, Maragakis NJ (2008) Focal transplantation-based astrocyte replacement is neuroprotective in a model of motor neuron disease. Nat Neurosci 11:1294-1301.

Levitt P, Harvey JA, Simansky K, Murphy EH (1997) New evidence for neurotransmitter influences on brain development. Trends Neurosci 20:269-274. 
Liu Z, Geng L, Li R, He X, Zheng JQ, Xie Z (2003) Frequency modulation of synchronized Ca2+ spikes in cultured hippocampal networks through G-proteincoupled receptors. J Neurosci 23:4156-4163.

Lusin JD, Vanarotti M, Li C, Valiveti A, Ames JB (2008) NMR structure of DREAM: Implications for $\mathrm{Ca}(2+)$-dependent $\mathrm{DNA}$ binding and protein dimerization. Biochemistry 47:2252-2264.

Mabie PC, Mehler MF, Kessler JA (1999) Multiple roles of bone morphogenetic protein signaling in the regulation of cortical cell number and phenotype. J Neurosci 19:7077-7088.

Maragakis NJ, Rothstein JD (2004) Glutamate transporters: animal models to neurologic disease. Neurobiol Dis 15:461-473.

Matallanas D, Arozarena I, Berciano M, Aaronson DS, Pellicer A, Lafarga M, Crespo P (2003) Differences on the inhibitory specificities of H-Ras, K-Ras, and N-Ras (N17) dominant negative mutants are related to their membrane microlocalization. J Biol Chem 278:4572-4581.

McManus M, Chen LC, Vallejo I, Vallejo M (1999) Astroglial differentiation of cortical precursor cells triggered by activation of the cAMP-dependent signaling pathway. J Neurosci 19:9004-9015.

Mehler MF, Mabie PC, Zhang D, Kessler JA (1997) Bone morphogenetic proteins in the nervous system. Trends Neurosci 20:309-317.

Miller FD, Gauthier AS (2007) Timing is everything: Making neurons versus glia in the developing cortex. Neuron 54:357-369.

Morita K, Sakakibara A, Kitayama S, Kumagai K, Tanne K, Dohi T (2002) Pituitary adenylate cyclase-activating polypeptide induces a sustained increase in intracellular free $\mathrm{Ca}^{2+}$ concentration and catecholamine release by activating 
$\mathrm{Ca}^{2+}$ influx via receptor-stimulated $\mathrm{Ca}^{2+}$ entry, Independent of store-operated $\mathrm{Ca}^{2+}$ channels, and voltage-dependent $\mathrm{Ca}^{2+}$ channels in bovine adrenal medullary chromaffin cells. J Pharmacol Exp Ther 302:972-982.

Mukhopadhyay SS, Wyszomierski SL, Gronostajski RM, Rosen JM (2001) Differential interactions of specific nuclear factor I isoforms with the glucocorticoid receptor and STAT5 in the cooperative regulation of WAP gene transcription. Mol Cell Biol 21:6859-6869.

Nakashima K, Yanagisawa M, Arakawa H, Kimura N, Hisatsune T, Kawabata M, Miyazono K, Taga T (1999a) Synergistic signaling in fetal brain by STAT3Smad1 complex bridged by p300. Science 284:479-482.

Nakashima K, Wiese S, Yanagisawa M, Arakawa H, Kimura N, Hisatsune T, Yoshida K, Kishimoto T, Sendtner M, Taga T (1999b) Developmental requirement of gp130 signaling in neuronal survival and astrocyte differentiation. J Neurosci 19:5429-5434.

Namihira M, Nakashima K, Taga T (2004) Developmental stage-dependent regulation of DNA methylation and chromatin modification in an immature astrocytespecific gene promoter. FEBS Lett 572:184-188.

Nedergaard M, Ransom B, Goldman SA (2003) New roles for astrocytes: Redefining the functional architecture of the brain.

Nicot A, DiCicco-Bloom E (2001) Regulation of neuroblast mitosis is determined by PACAP receptor isoform expression. Proc Natl Acad Sci USA 98:4758-4763.

Nieto M, Schuurmans C, Britz O, Guillemot F (2001) Neural bHLH genes control the neuronal versus glial fate decision in cortical progenitors. Neuron 29:401-413. 
Nishimoto M, Furuta A, Aoki S, Kudo Y, Miyakawa H, Wada K (2007) PACAP/PAC1 autocrine system promotes proliferation and astrogenesis in neural progenitor cells. Glia 55:317-327.

Obara Y, Horgan A, Stork PJS (2007) The requirement of Ras and Rap1 for the activation of ERKs by cAMP, PACAP, and $\mathrm{KCl}$ in cerebellar granule cells. $\mathrm{J}$ Neurochem 101:470-482.

Ohno F, Watanabe J, Sekihara H, Hirabayashi T, Arata S, Kikuyama S, Shioda S, Nakaya K, Nakajo S (2005) Pituitary adenylate cyclase-activating polypeptide promotes differentiation of mouse neural stem cells into astrocytes. Regul Pept 126:115-122.

Osawa M, Dace A, Tong KI, Valiveti A, Ikura M, Ames JB (2005) Mg2+ and Ca2+ differentially regulate DNA binding and dimerization of DREAM. J Biol Chem 280:18008-18014.

Osawa M, Tong KI, Lilliehook C, Wasco W, Buxbaum JD, Cheng HYM, Penninger JM, Ikura M, Ames JB (2001) Calcium-regulated DNA binding and oligomerization of the neuronal calcium-sensing protein, calsenilin/DREAM/KChIP3. J Biol Chem 276:41005-41013.

Osipenko ON, Barrie A, Allen JM, Gurney AM (2000) Pituitary adenylate cyclaseactivating peptide activates multiple intracellular signaling pathways to regulate ion channels in PC12 cells. J Biol Chem 275:16626-16631.

Park JK, Williams BP, Alberta JA, Stiles CD (1999) Bipotent cortical progenitor cells process conflicting cues for neurons and glia in a hierarchical manner. J Neurosci 19:10383-10389.

Payet M, Bilodeau L, Breault L, Fournier A, Yon L, Vaudry H, Gallo-Payet N (2003) $\mathrm{PAC}_{1}$ receptor activation by PACAP-38 mediates $\mathrm{Ca}^{2+}$ release from a cAMP- 
dependent pool in human fetal adrenal gland chromaffin cells. J Biol Chem 278:1663-1670.

Perea G, Araque A (2007) Astrocytes potentiate transmitter release at single hippocampal synapses. Science 317:1083-1086.

Pham N, Cheglakov I, Koch CA, de Hoog CL, Moran MF, Rotin D (2000) The guanine nucleotide exchange factor CNrasGEF activates Ras in response to cAMP and cGMP. Curr Biol 10:555-558.

Pinto L, Gotz M (2007) Radial glial cell heterogeneity - The source of diverse progeny in the CNS. Prog Neurobiol 83:2-23.

Przywara DA, Guo X, Angelilli ML, Wakade TD, Wakade AR (1996) A noncholinergic transmitter, pituitary adenylate cyclase-activating polypeptide, utilizes a novel mechanism to evoke catecholamine secretion in rat adrenal chromaffin cells. J Biol Chem 271:10545-10550.

Qian X, Davis AA, Goderie SK, Temple S (1997) FGF2 concentration regulates the generation of neurons and glia from multipotent cortical stem cells. Neuron 18:81-93.

Quinlan RA, Brenner M, Goldman JE, Messing A (2007) GFAP and its role in Alexander disease. Exp Cell Res 10:2077-2087.

Rajan P, McKay RDG (1998) Multiple routes to astrocytic differentiation in the CNS. J Neurosci 18:3620-3629.

Rajan P, Panchision DM, Newell LF, McKay RDG (2003) BMPs signal alternately through a SMAD or FRAP-STAT pathway to regulate fate choice in CNS stem cells. J Cell Biol 161:911-921. 
Rivas M, Mellstrom B, Naranjo JR, Santisteban P (2004) Transcriptional repressor DREAM interacts with thyroid transcription factor-1 and regulates thyroglobulin gene expression. J Biol Chem 279:33114-33122.

Romano D, Magalon K, Ciampini A, Talet C, Enjalbert A, Gerard C (2003) Differential involvement of the Ras and Rap1 small GTPases in vasoactive intestinal and pituitary adenylyl cyclase activating polypeptides control of the prolactin gene. $\mathrm{J}$ Biol Chem 278:51386-51394.

Schindler C, Levy DE, Decker T (2007) JAK-STAT signaling: From interferons to cytokines. J Biol Chem 282:20059-20063.

Scsucova S, Palacios D, Savignac M, Mellstrom B, Naranjo JR, Aranda A (2005) The repressor DREAM acts as a transcriptional activator on vitamin D and retinoic acid response elements. Nucl Acids Res 33:2269-2279.

Seri B, García-Verdugo JM, McEwen BS, Alvarez-Buylla A (2001) Astrocytes give rise to new neurons in the adult mammalian hippocampus. J Neurosci 21:7153-7160.

Shu T, Butz KG, Plachez C, Gronostajski RM, Richards LJ (2003) Abnormal development of forebrain midline glia and commissural projections in Nfia knock-out mice. J Neurosci 23:203-212.

Song MR, Ghosh A (2004) FGF2-induced chromatin remodeling regulates CNTFmediated gene expression and astrocyte differentiation. Nat Neurosci 7:229-235.

Spengler D, Waeber C, Pantaloni C, Florian H, Bockaert J, Seeburg PH, Journot L (1993) Differential signal transduction by five splice variants of the PACAP receptor. Nature 365:170-175.

Steele-Perkins G, Plachez C, Butz KG, Yang G, Bachurski CJ, Kinsman SL, Litwack ED, Richards LJ, Gronostajski RM (2005) The transcription factor gene Nfib is essential for both lung maturation and brain development. Mol Cell Biol 25:685. 
Stone DJ, Song Y, Anderson CP, Krohn KK, Finch CE, Rozovsky I (1998) Bidirectional transcription regulation of glial fibrillary acidic protein by estradiol in vivo and in vitro. Endocrinology 139:3202-3209.

Suh J, Lu N, Nicot A, Tatsuno I, DiCicco-Bloom E (2001) PACAP is an anti-mitogenic signal in developing cerebral cortex. Nat Neurosci 4:123-124.

Sun Y, Nadal-Vicens M, Misono S, Lin MZ, Zubiaga A, Hua X, Fan G, Greenberg ME (2001) Neurogenin promotes neurogenesis and inhibits glial differentiation by independent mechanisms. Cell 104:365-376.

Takizawa T, Nakashima K, Namihira M, Ochiai W, Uemura A, Yanagisawa M, Fujita N, Nakao M, Taga T (2001) DNA methylation is a critical cell-intrinsic determinant of astrocyte differentiation in the fetal brain. Dev Cell 1:749-758.

Theodosis DT, Poulain DA, Oliet SHR (2008) Activity-dependent structural and functional plasticity of astrocyte-neuron interactions. Physiol Rev 88:983-1008.

Vallejo I, Vallejo M (2002) Pituitary adenylate cyclase-activating polypeptide induces astrocyte differentiation of precursor cells from developing cerebral cortex. Mol Cell Neurosci 21:671-683.

Viti J, Feathers A, Phillips J, Lillien L (2003) Epidermal growth factor receptors control competence to interpret leukemia inhibitory factor as an astrocyte inducer in developing cortex. J Neurosci 23:3385-3393.

Vossler MR, Yao H, York R, Pan MG, Rim CS, Stork PJS (1997) cAMP activates MAP kinase and Elk-1 through a B-Raf- and Rap1-Dependent pathway. Cell 89:7382.

Watanabe J, Ohba M, Ohno F, Kikuyama S, Nakamura M, Nakaya K, Arimura A, Shioda S, Nakajo S (2006) Pituitary adenylate cyclase-activating polypeptide- 
induced differentiation of embryonic neural stem cells into astrocytes is mediated via the $\beta$ isoform of protein kinase C. J Neurosci Res 84:1645-1655.

Watanabe J, Nakamachi T, Matsuo R, Hayashi D, Nakamura M, Kikuyama S, Nakago S, Shioda S (2007) Localization, characterization and function of pituitary adenylate cyclase-activating polypeptide during brain developent. Peptides 28:1713-1719.

Williams BP, Park JK, Alberta JA, Muhlebach SG, Hwang GY, Roberts TM, Stiles CD (1997) A PDGF-regulated immediate early gene response initiates neuronal differentiation in ventricular zone progenitor cells. Neuron 18:553-552.

Zupan V, Hill JM, Brenneman DE, Gozes I, Fridkin M, Roberecht P, Evrard P, Gressens P (1998) Involvement of pituitary adenylate cyclase-activating polypeptide II vasoactive intestinal peptide 2 receptor in mouse neocortical astrocytogenesis. J Neurochem 70:2165-2173. 


\section{FIGURE LEGENDS:}

Figure 1. Schematic depiction of a simplified model to illustrate a possible role DREAM in the regulation of GFAP gene transcription. A) In a normal situation, DREAM is bound to a site located in close proximity to the site bound by NFI, so that it is possible that DREAM and NFI interact. In addition, it is possible that DREAM and NFI interact with other unidentified transcription factors (UTF) that are required for transcriptional activity (arrow 1). DREAM mediates transcriptional responses to PACAP/cAMP (arrow 2), whereas STAT proteins, which bind to promoter elements located upstream, mediate transcriptional responses to neurotrophic cytokines such as CNTF or cardiotrophin-1 (arrow 3). In addition, NFI binding is required for the synergistic cooperation between CNTF and PACAP, which may involve transcriptional interactions (arrow 4). B) Sequestration of NFI and possibly other UTF by DREAM would explain that mutation of the binding sites for DREAM abolish basal and stimulated activity of the GFAP promoter. C) In DREAM deficient mice, PACAPdependent stimulation of GFAP transcription is inhibited, but the binding of other transcriptional transactivators remains unaltered (arrow 1) and gp130-JAK-STATinduced promoter activity is intact (arrow 3). In vivo, this results in delayed astrogliogenesis as a result of the absence of the PACAP-DREAM-dependent signal. D) When the NFI binding site is mutated, stimulation of the gp130-JAK-STAT and PACAP-DREAM signaling pathways are intact (arrows 2 and 3), but synergistic cooperation cannot occur (arrow 4) and overall promoter activity is decreased.

Figure 2. Schematic depiction of the cAMP/calcium-dependent pathway activated by PACAP during astrocyte differentiation. PACAP acts on cortical precursors by activating seven transmembrane spanning domain PAC1 receptors. This results in the 
stimulation of intracellular cAMP production by G-protein-mediated stimulation of adenylate cyclase (AC). Cyclic AMP activates PKA, Epac-Rap1 and Ras (probably by acting on CNrasGEF), but PKA does not contribute to stimulation of GFAP gene transcription during astrocyte differentiation. Exposure of precursor cells to PACAP results in calcium entry from the extracellular milieu, an effect that requires the activity of cAMP. Finally, calcium binds to DREAM, which occupies specific sites on the GFAP promoter, and following this GFAP gene transcription is stimulated. Independently of this pathway, GFAP transcription can also be stimulated by neurotrophic cytokines such as CNTF or cardiotrophin-1 (CT-1) via activation of the gp130-JAK-STAT pathway. Direct or indirect interactions between STAT proteins and DREAM, as described in Figure 1, could explain synergistic interactions of both pathways at the transcriptional level. 


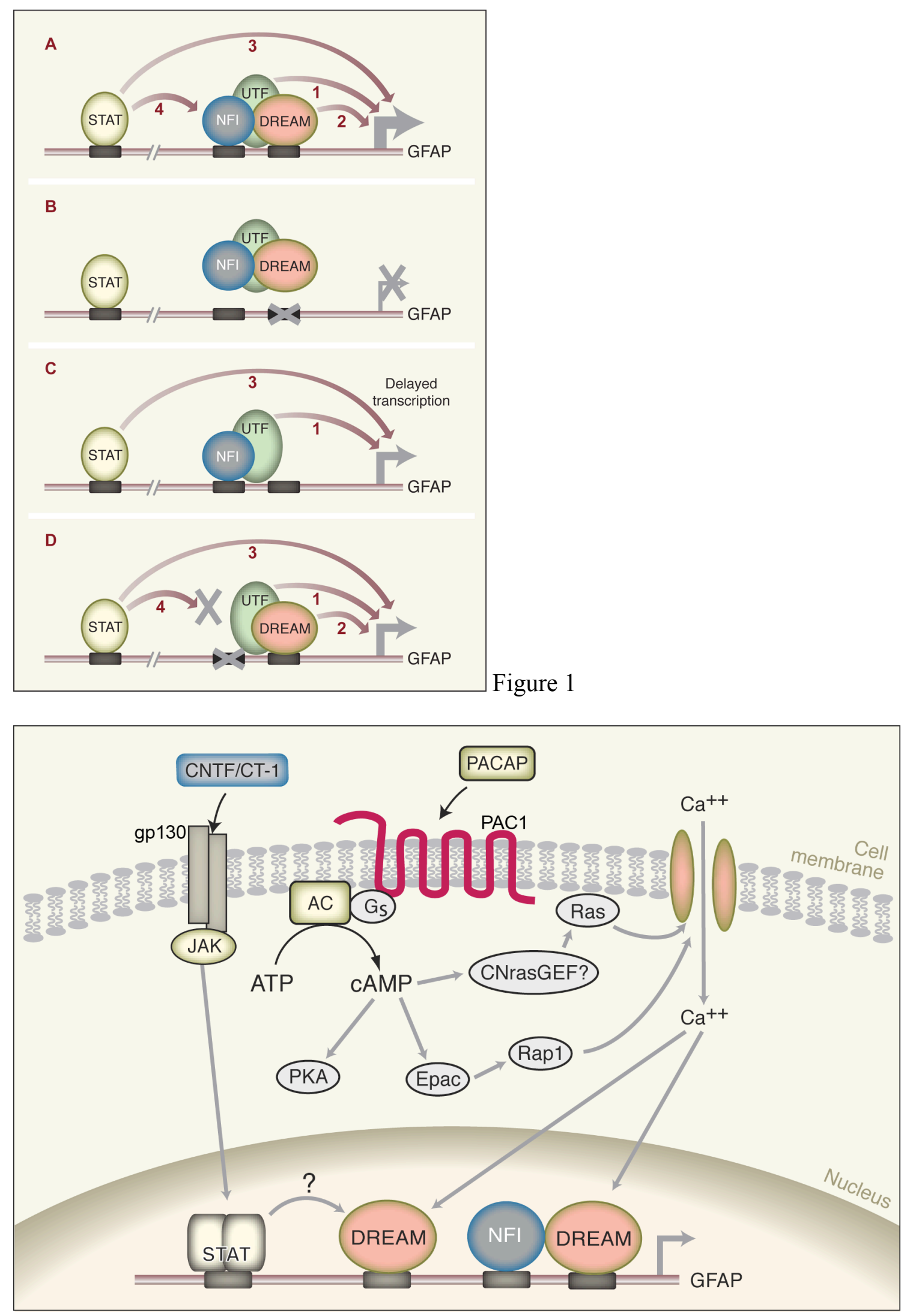

Figure 2 